\title{
INFLUÊNCIA DA ATMOSFERA NA DEGRADAÇÃO TÉRMICA OXIDATIVA DE GRAXAS LUBRIFICANTES À BASE DE CÁLCIO
}

\author{
L. M. R. LIMA' ${ }^{1}$, A. G. de SOUZA 2 , J. C. O. SANTOS ${ }^{3}$, J. C. A. da SILVA \\ ${ }^{1}$ Universidade Federal de Campina Grande, Unidade Acadêmica de Tecnologia do \\ Desenvolvimento \\ ${ }^{2}$ Universidade Federal da Paraíba, Departamento de Química \\ ${ }^{3}$ Universidade Federal de Campina Grande, Unidade Acadêmica de Química \\ ${ }^{4}$ Embrapa Algodão, Campina Grande - PB \\ E-mail para contato: mergia@ufcg.edu.br
}

\begin{abstract}
RESUMO - Uma das principais vantagens das graxas lubrificantes é o fato de não escorrerem do local em que são colocadas, o que dispensa a implantação de sistemas dispendiosos de vedação. A degradação das graxas sob condições de operação ocorre principalmente através de mecanismos de oxidação. O objetivo deste trabalho foi determinar a influência da atmosfera na degradação térmica oxidativa de graxas lubrificantes à base de cálcio. Para tanto, as amostras foram submetidas a diferentes temperaturas $\left(150,170,190\right.$ e $\left.210{ }^{\circ} \mathrm{C}\right)$, durante períodos de tempo distintos ( 1 e $6 \mathrm{~h}$ ), sendo caracterizadas por técnicas de análise térmica, em atmosfera inerte $\left(\mathrm{N}_{2}\right)$ e oxidante $\left(\mathrm{O}_{2}\right)$, e espectroscopia na região do infravermelho. Os resultados mostraram que, em atmosfera oxidante, houve deslocamento das curvas TG para temperaturas mais baixas, provavelmente devido ao fato das amostras terem sofrido combustão. Em atmosfera inerte, a decomposição ocorreu em número maior de etapas do que em atmosfera oxidante, gerando menor quantidade de resíduos do que as amostras submetidas à atmosfera de $\mathrm{O}_{2}$. Além disso, houve pequenas modificações nas temperaturas de início de decomposição das amostras degradadas em comparação com as não degradadas. Com relação ao Infravermelho, os espectros apontaram que a degradação ocorreu através de processo de oxidação, resultando na formação de compostos carbonilados, confirmados pela presença de bandas referentes aos grupos $\mathrm{C}=\mathrm{O}$ e $\mathrm{C}-\mathrm{C}(\mathrm{C}=\mathrm{O})-\mathrm{O}$.
\end{abstract}

\section{INTRODUÇÃO}

A lubrificação consiste da separação de dois elementos mecânicos em movimento, o que gera atrito e desgaste. Os lubrificantes formam um filme fino de óleo evitando contatos metálicos diretos (RUPRECHT, 2008).

O lubrificante é um fluido que, aplicado às máquinas, cria uma camada impermeável entre as peças, reduz o aquecimento e evita que aquelas em movimento provoquem atrito ou se desgastem. É um material mole e facilmente deformável que apresenta ligações secundárias fracas. 
Graxa lubrificante é um produto sólido ou semissólido, consistindo de um agente espessante e um líquido lubrificante. Graxas apropriadas resolvem problemas de lubrificação sem corrosão, mesmo em presença de água. São lubrificantes feitos à base de um sabão metálico, geralmente de lítio, cálcio ou sódio enriquecido às vezes com aditivos de grafite, molibdênio, entre outros. As graxas devem possuir boa adesividade e resistência ao trabalho, além de suportarem bem ao calor e à ação da água e umidade (RUPRECHT, 2008).

A vida útil de um equipamento com partes sólidas em contato depende, em grande parte, da eficiência de lubrificação da graxa utilizada. Com isso, inúmeros estudos têm sido desenvolvidos para avaliar as propriedades de resistência das graxas lubrificantes, podendo se citar: Czarny (1995); Yonggang e Jie (1998); Hsu et al. (2004); Yeong et al. (2004); Lima et al. (2007) e Cavalcante (2012).

As graxas à base de sabão de cálcio apresentam estrutura macia, similar a da manteiga, e boa estabilidade mecânica. São normalmente estáveis com 1 a $3 \%$ de água e não são solúveis em água. São recomendadas para instalações expostas à água, tais como seção úmida de máquinas de fabricação de papel. Proporcionam boa proteção contra água salina e podem ser utilizadas com segurança em ambientes marinhos (LUBRIFICAÇÃO..., 2008).

De acordo com Antoniassi (2001), a estabilidade oxidativa é definida como a resistência da amostra à oxidação. É expressa pelo período de indução - tempo entre o início da medição e o momento em que ocorre um aumento brusco na formação de produtos da oxidação —, que é dado em horas. Rosenhaim et al. (2006) destacam que a estabilidade oxidativa de uma amostra é um parâmetro de sua qualidade, estando relacionada ao tempo e às condições de armazenamento da mesma. Quanto mais resistente à oxidação for um lubrificante, menor será a tendência a formar depósitos e produtos corrosivos na graxa (SHARMA e STIPANOVIC, 2003).

Segundo Karacan et al. (1999), o processo de degradação térmica de um lubrificante ocorre através de dois mecanismos: oxidação e decomposição térmica. Os lubrificantes à base de óleos minerais apresentam natureza complexa devido à presença de uma grande variedade de grupos funcionais. Tal estrutura torna as reações de oxidação extremamente complexas e de difícil entendimento. É comumente aceito que a oxidação ocorre via mecanismo radical livre. Por outro lado, a decomposição térmica ocorre através da cisão das ligações $\mathrm{C}-\mathrm{C}$.

O objetivo principal deste trabalho foi estudar o processo de degradação térmica oxidativa de graxas lubrificantes à base de sabão de cálcio, avaliando a influência da razão de aquecimento no processo de decomposição.

\section{MATERIAIS E MÉTODOS}

Neste trabalho foi utilizada graxa lubrificante à base de cálcio, produzida por indústrias brasileiras e adquirida no comércio local. De acordo com o fabricante da graxa, trata-se de um produto contendo óleo lubrificante de alta viscosidade, com excelente bombeabilidade e manuseio, com alta adesão e oferecendo boa proteção contra corrosão e oxidação, indicada para uso automotivo, industrial, agrícola e uso geral. 
A degradação térmica das graxas lubrificantes foi realizada em condições de uso, sob atmosfera de ar, com temperaturas de $150,170,190$ e $210^{\circ} \mathrm{C}$, durante dois períodos de tempo distintos: 1 e 6 horas.

As graxas lubrificantes foram submetidas à análise, na forma encontrada para venda (sem degradação) e após a degradação térmica, a qual foi realizada em simulações de condições de uso, sob programas de tempo e temperatura pré-definidos de acordo com Santos et al. (2005), conforme mostra a Figura 1.

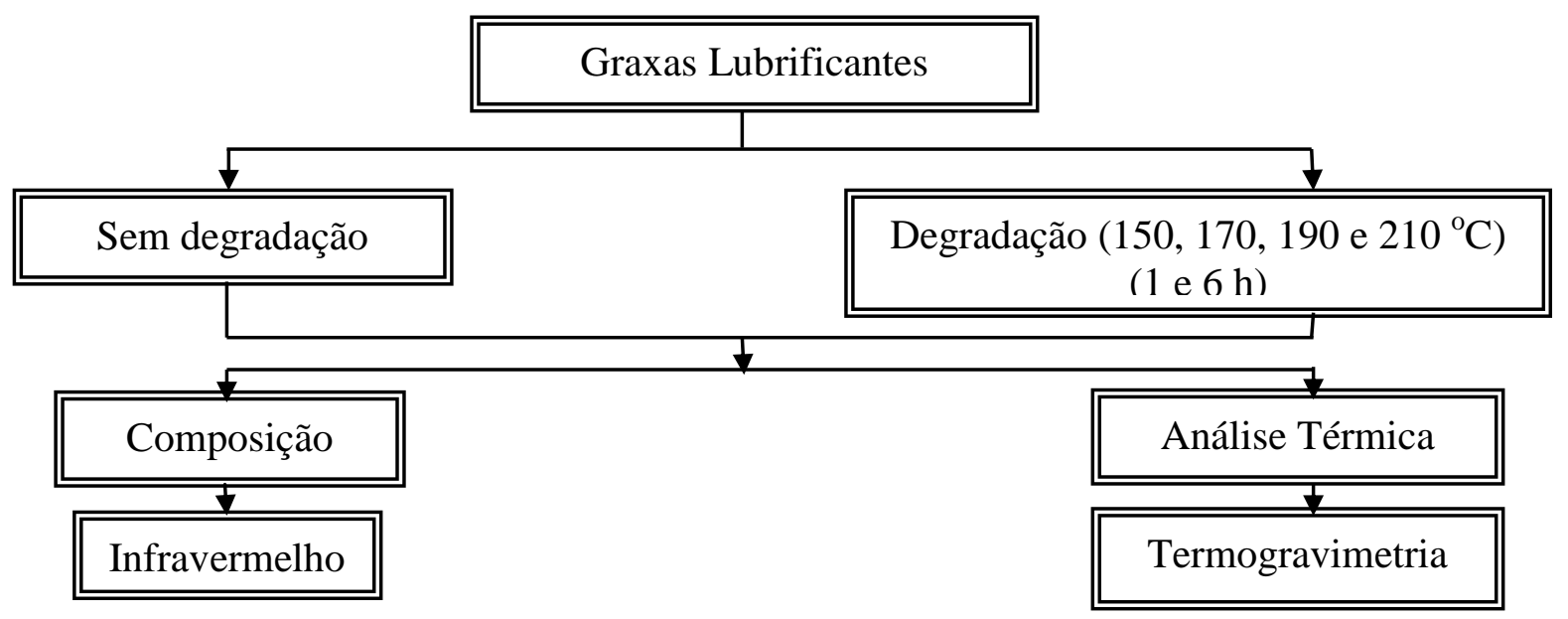

Figura 1 - Fluxograma da metodologia utilizada.

\section{RESULTADOS E DISCUSSÃO}

Antes de submeter as amostras à análise térmica, foi realizada a caracterização através da Espectroscopia na Região do Infravermelho. A espectroscopia na região do infravermelho tornou-se ferramenta importante em termos de análise de graxas. A técnica é particularmente benéfica para a compreensão de composições químicas complexas, para diagnósticos no caso de falha de lubrificação ou do lubrificante e monitoramento do desempenho de aditivos durante o funcionamento.

Os espectros de absorção na região do infravermelho foram obtidos em espectrômetro marca BOMEM, modelo MB-102, usando pastilhas de brometo de potássio, na faixa de 4000 $-400 \mathrm{~cm}^{-1}$.

\subsection{Espectroscopia de Absorção na Região do Infravermelho}

A técnica de espectroscopia de absorção na região do infravermelho foi aplicada para a investigação da composição das graxas lubrificantes, bem como dos compostos formados em decorrência do processo de degradação térmica oxidativa das amostras. 
Os espectros das amostras de graxa lubrificante à base de sabão de cálcio estudadas, antes da degradação e submetidas à degradação durante 1 h (a) e 6 h (b), nas temperaturas previamente indicadas podem ser observados conforme mostra a Figura 2.

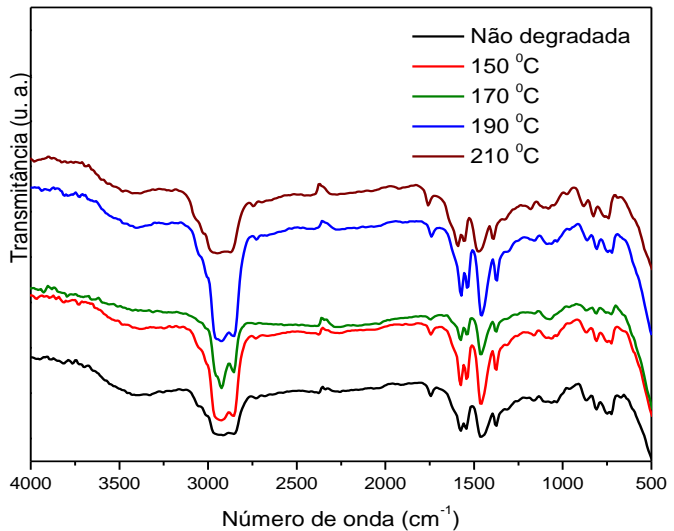

(a)

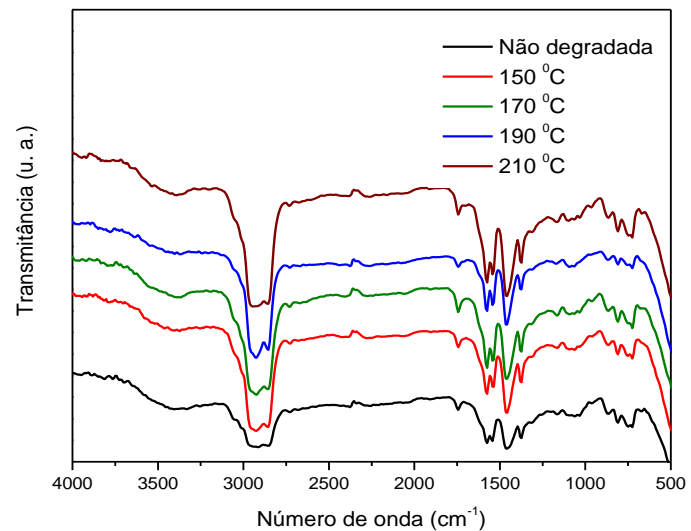

(b)

Figura 2 - Espectros de absorção na região do infravermelho da graxa lubrificante à base de sabão de cálcio não degradada e submetida à degradação a diferentes temperaturas durante 1 hora (a) e 6 horas (b).

Os espectros da Figura 2a, para a amostra submetida à degradação durante 1 hora, apresentam bandas fracas e largas a aproximadamente $3400 \mathrm{~cm}^{-1}$, para todas as amostras (não degradada e submetidas à degradação nas diferentes temperaturas de estudo), relativas provavelmente à presença de grupamentos $\mathrm{O}$ - H (SILVERSTEIN e WEBSTER, 2006).

$\mathrm{Na}$ região de 2900 a $2800 \mathrm{~cm}^{-1}$, os espectros apresentam bandas características de vibrações de deformação axial de $\mathrm{C}-\mathrm{H}$ de grupos $\mathrm{CH}_{2}$ e $\mathrm{CH}_{3}$ de alcanos, as quais sofreram apenas modificações na intensidade para as amostras degradadas, devido possivelmente a modificações sofridas na estrutura da graxa lubrificante com a formação de novos compostos.

Podem ser observadas bandas características na região de $1750 \mathrm{~cm}^{-1}$, provavelmente referentes à vibração de deformação axial da carbonila $(\mathrm{C}=\mathrm{O})$ de ésteres, tanto no perfil da curva da graxa não degradada, quanto naqueles relativos às amostras degradadas nas quatro temperaturas de trabalho, que são corroboradas por Silverstein e Webster (2006). A pequena intensidade destas bandas pode ser justificada pela influência dos grupos participantes da composição da graxa lubrificante, que apresenta aditivos que afetam a frequência em que a banda característica surge. Além disso, existe a presença de um metal alcalino que, de certa forma, irá influenciar no espectro da amostra estudada.

A região de 1600 a $1300 \mathrm{~cm}^{-1}$ apresenta bandas características da deformação angular de grupos $\mathrm{CH}_{3}$ presentes em cetonas e ésteres. A presença de cetonas e ésteres conduz ao aparecimento de deformações axial e angular do tipo $\mathrm{C}-\mathrm{C}(\mathrm{C}=\mathrm{O})-\mathrm{O}$.

As bandas apresentadas nos espectros da Figura $2 \mathrm{~b}$ mostram que não houve mudança nas amostras com relação ao tempo de degradação no que diz respeito aos seus espectros na região de infravermelho. Foram detectadas as seguintes vibrações: bandas fracas e largas a aproximadamente $3400 \mathrm{~cm}^{-1}$, referentes provavelmente à presença de grupamentos $\mathrm{O}-\mathrm{H}$ 
impedidos ou participantes de ligações hidrogênio; bandas características de vibrações de deformação axial de $\mathrm{C}-\mathrm{H}$ de grupos $\mathrm{CH}_{2}$ e $\mathrm{CH}_{3}$ de alcanos na região de 2900 a $2800 \mathrm{~cm}^{-1}$, na região de $1750 \mathrm{~cm}^{-1}$, referentes à vibração da carbonila $(\mathrm{C}=\mathrm{O})$ de ácidos carboxílicos ou aldeídos gerados como produtos de oxidação; na região de 1600 a $1300 \mathrm{~cm}^{-1}$, bandas características da deformação angular de grupos $\mathrm{CH}_{3}$ presentes em cetonas e ésteres, levando ao surgimento de deformações axial e angular do tipo $\mathrm{C}-\mathrm{C}(\mathrm{C}=\mathrm{O})-\mathrm{O}$.

\subsection{Análise Térmica}

Termogravimetria (TG): A termogravimetria foi utilizada para estudar o comportamento térmico das graxas, relacionado à sua degradação térmica, quando as amostras foram submetidas a temperaturas desde a ambiente até $750{ }^{\circ} \mathrm{C}$, bem como para determinar sua estabilidade térmica.

As curvas TG/DTG das amostras (não degradadas e degradadas) foram obtidas em dois equipamentos de análise térmica. Sob atmosfera inerte de nitrogênio $\left(\mathrm{N}_{2}\right)$, foi utilizado um analisador térmico simultâneo, marca TA Instruments, modelo SDT-2960, com fluxo de 110 $\mathrm{mL} \cdot \mathrm{min}^{-1}$, nas razões de aquecimento de 10 e $20^{\circ} \mathrm{C} \mathrm{min}^{-1}$, massa em torno de $10,0 \pm 0,5 \mathrm{mg}$, intervalo da temperatura ambiente a $750{ }^{\circ} \mathrm{C}$ e utilizando-se cadinhos de alumina. Sob atmosfera oxidante de oxigênio $\left(\mathrm{O}_{2}\right)$, foi utilizada uma termobalança da marca SHIMADZU, modelo TGA-50, com fluxo de $50 \mathrm{~mL} \cdot \mathrm{min}^{-1}$, nas razões de aquecimento de 10 e $20{ }^{\circ} \mathrm{C} \mathrm{min}^{-1}$, massa em torno de $5,0 \pm 0,5 \mathrm{mg}$, intervalo de temperatura de 25 a $750{ }^{\circ} \mathrm{C}$, utilizando-se cadinhos de alumina.

Conforme mostra a Figura 3, podem-se observar as curvas termogravimétricas das graxas lubrificantes à base de sabão de cálcio não degradadas, a razões de aquecimento de 10 e $20{ }^{\circ} \mathrm{C} \cdot \mathrm{min}^{-1}$, respectivamente, em atmosferas de nitrogênio e oxigênio.

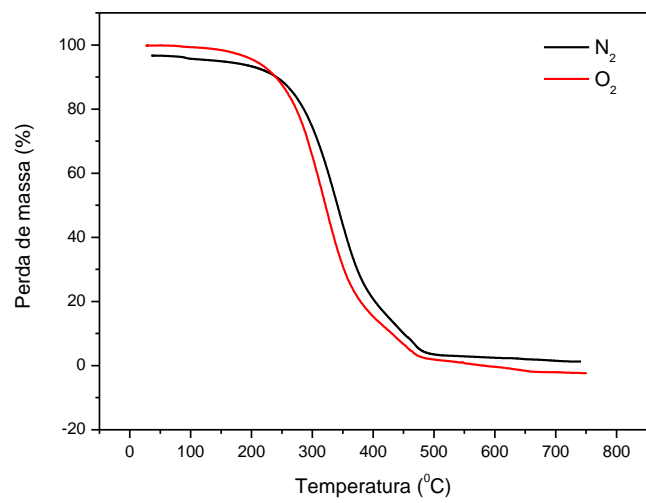

(a)

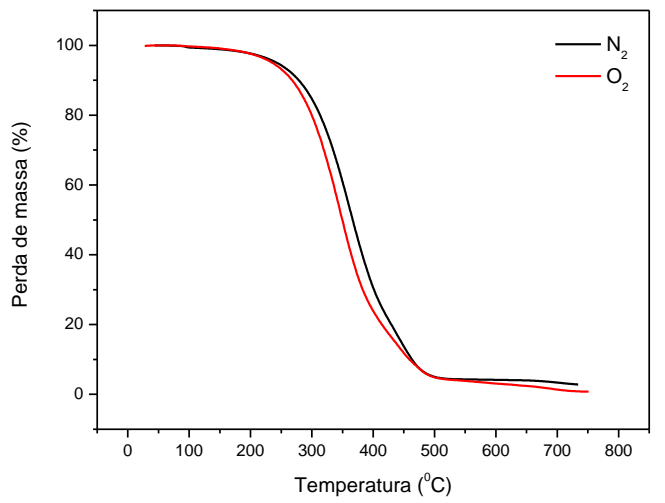

(b)

Figura 3 - Curvas TG das amostras de graxa à base de sabão de cálcio não degradadas, em atmosferas de nitrogênio e de oxigênio: (a) À razão de aquecimento de $10{ }^{\circ} \mathrm{C} \cdot \mathrm{min}^{-1}$; (b) À razão de aquecimento de $20^{\circ} \mathrm{C} \cdot \mathrm{min}^{-1}$. 
De acordo com as curvas da Figura 3a, observa-se que, em atmosfera de oxigênio, houve um pequeno deslocamento das curvas para temperaturas mais baixas, o que pode ser atribuído ao favorecimento do processo de decomposição.

Com relação ao número de etapas de decomposição, observa-se que, em atmosfera de nitrogênio, de forma geral a decomposição ocorreu em número maior de etapas do que em atmosfera de oxigênio.

A perda inicial de massa maior foi observada em atmosfera inerte (nitrogênio). No que se referem aos resíduos da decomposição, as amostras submetidas à atmosfera de nitrogênio produziram quantidade menor de resíduo do que aquelas submetidas à atmosfera de oxigênio.

As curvas das amostras de graxa à base de sabão de cálcio obtidas à razão de aquecimento de $20{ }^{\circ} \mathrm{C} \cdot \mathrm{min}^{-1}$ (Figura $3 \mathrm{~b}$ ) apresentaram perfil semelhante àquelas obtidas à razão de $10{ }^{\circ} \mathrm{C} \cdot \mathrm{min}^{-1}$, tanto em atmosfera inerte (nitrogênio) quanto em atmosfera oxidante (oxigênio).

Conforme mostra a Figura 4, podem-se observar as curvas termogravimétricas das graxas lubrificantes à base de sabão de cálcio submetidas à degradação na temperatura de 150 ${ }^{\circ} \mathrm{C}$, a razões de aquecimento de 10 e $20{ }^{\circ} \mathrm{C} \cdot \mathrm{min}^{-1}$, respectivamente, em atmosferas de nitrogênio e oxigênio.

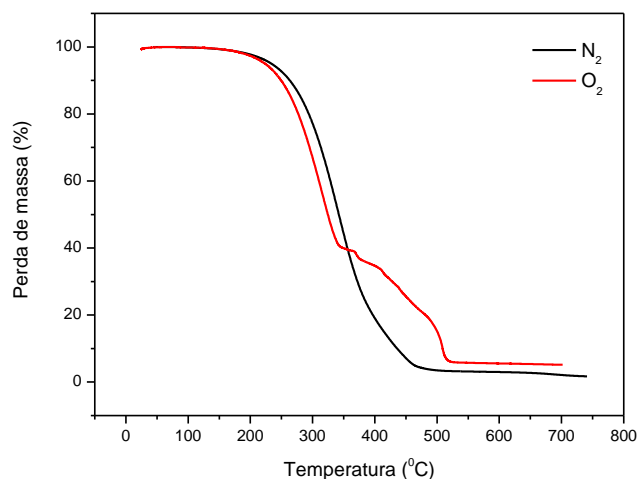

(a)

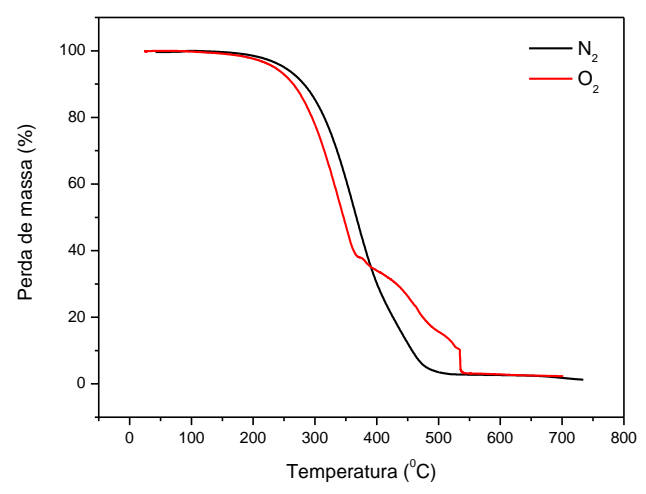

(b)

Figura 4 - Curvas TG das amostras de graxa à base de sabão de cálcio submetida à degradação a $150{ }^{\circ} \mathrm{C}$, em atmosferas de nitrogênio e de oxigênio: (a) À razão de aquecimento de $10{ }^{\circ} \mathrm{C} \cdot \mathrm{min}^{-1}$; (b) À razão de aquecimento de $20{ }^{\circ} \mathrm{C} \cdot \mathrm{min}^{-1}$.

As curvas das amostras de graxa à base de sabão de cálcio submetidas à degradação a $150{ }^{\circ} \mathrm{C}$, obtidas às duas razões de aquecimento, apresentaram comportamento semelhante em atmosfera inerte (nitrogênio). Em atmosfera de oxigênio, a aproximadamente $500{ }^{\circ} \mathrm{C}$, surgiu o comportamento referente à combustão da amostra, apresentando um perfil que diferenciou-se daquele apresentado pelas curvas em atmosfera inerte. 
Conforme mostra a Figura 5, podem-se observar as curvas TG das graxas lubrificantes à base de sabão de cálcio submetidas à degradação na temperatura de $210{ }^{\circ} \mathrm{C}$, a razões de aquecimento de 10 e $20{ }^{\circ} \mathrm{C} \cdot \mathrm{min}^{-1}$, respectivamente, em atmosferas de nitrogênio e oxigênio.

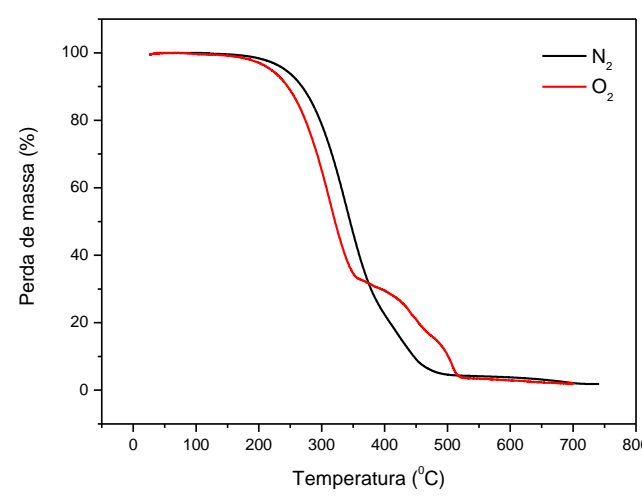

(a)

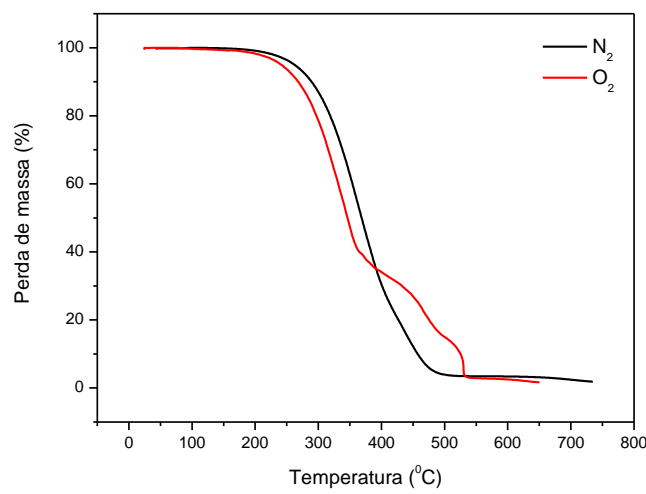

(b)

Figura 5 - Curvas TG das amostras de graxa à base de sabão de cálcio submetida à degradação a $210{ }^{\circ} \mathrm{C}$, em atmosferas de nitrogênio e de oxigênio: (a) À razão de aquecimento de $10{ }^{\circ} \mathrm{C} \cdot \mathrm{min}^{-1}$; (b) À razão de aquecimento de $20{ }^{\circ} \mathrm{C} \cdot \mathrm{min}^{-1}$.

As curvas termogravimétricas das amostras de graxa à base de sabão de cálcio submetidas à degradação a $210{ }^{\circ} \mathrm{C}$, obtidas às duas razões de aquecimento, apresentaram comportamento semelhante àquelas obtidas à temperatura de $150{ }^{\circ} \mathrm{C}$ : em atmosfera de oxidante, a aproximadamente $500{ }^{\circ} \mathrm{C}$, surgiu o comportamento referente à combustão da amostra.

\section{CONCLUSÕES}

A partir dos resultados obtidos, podem-se admitir as seguintes conclusões:

- Os espectros na região do infravermelho apontaram que a degradação das graxas lubrificantes estudadas ocorreu através de processo de oxidação, resultando na formação de compostos carbonilados.

- O estudo de análise térmica mostrou que houve pequenas modificações na temperatura de início de decomposição das amostras degradadas com relação às não degradadas.

- Em atmosfera de oxigênio, com relação à atmosfera inerte $\left(\mathrm{N}_{2}\right)$, houve modificação no perfil das curvas TG, provavelmente devido ao fato das amostras terem sofrido combustão.

- Com relação ao número de etapas de decomposição, em atmosfera de nitrogênio, normalmente a decomposição ocorreu em número maior de etapas do que em atmosfera de oxigênio. 
- No que se referem aos resíduos da decomposição, as amostras submetidas à atmosfera de nitrogênio produziram quantidade menor de resíduo do que aquelas submetidas à atmosfera de oxigênio.

\section{REFERENCIAS}

ANTONIASSI, R. Métodos de avaliação da estabilidade oxidativa de óleos e gorduras. Boletim do Centro de Pesquisa e Processamento de Alimentos (CEPPA), v. 19, n. 2, 353-380, Curitiba, jul./dez 2001.

CAVALCANTE, I. E. M. Estudo cinético e reológico de graxas lubrificantes submetidas à degradação térmica oxidativa, Dissertação (Mestrado), Universidade Federal da Paraíba, 2012.

CZARNY, R. Effect of changes in grease structure on sliding friction, Industrial Lubrication and Tribology, Vol. 47 No. 1, MCB University Press, 0036-8792, ILT, January/February, 1995.

HSU, A.; JONES, K. C.; FOGLIA, T. A.; MARMER, W. N. Continuous production of ethyl esters of grease using an immobilized lipase, JAOCS, Vol. 81, $\mathrm{N}^{0}$ 8, 2004.

KARACAN, Ö.; KÖK, M. V.; KARAASLAN, U. Dependence of thermal stability of an engine lubricating oil on usage period, J. of Therm. Analy. and Cal., Vol. 55, 109-114, 1999.

LIMA, L. M. R., SANTOS, J. C. O.; SOUZA, A. G. Thermal and kinetic study of lubricating Lithium Greases, J. of Eng. and App. Sci., 2(4); 718-721, 2007.

LUBRIFICAÇÃO, Disponível em: www.lubrificantes.net, Acesso em: 23 de Julho de 2008.

ROSENHAIM, R.; TAVARES, M. L. A.; CONCEIÇÃO, M. M.; LIMA, A. E. A.; SANTOS, I. M. G.; SOUZA, A. G. Estudo por termogravimetria do biodiesel de origem de óleos vegetais usados, misturas B5, B10, B15, B20 e B25 e diesel mineral. In: Congresso da Rede Brasileira de Tecnologia de Biodiesel, Livro de Resumos, Rede Brasileira de Tecnologia de Biodiesel, v. 1, p. 181-185, Brasília, 2006.

RUPRECHT, V. C. Lubrificação, Fricção e Adesão, Disponível em: www.uergs.edu.br, Acesso em: 26 de Novembro de 2008.

SHARMA, B. K.; STIPANOVIC, A. J. Development of a new oxidation stability test method for lubricating oils using high-pressure differential scanning calorimetry, Therm. Acta, 402, 1-18, 2003.

SILVERSTEIN, R. M.; WEBSTER, F. X. Identificação Espectrométrica de Compostos Orgânicos, Rio de Janeiro: LTC - Livros Técnicos e Científicos Editora S. A., 2006.

YEONG, S. K.; LUCKHAM, P. F.; TADROS, Th. F. Steady flow and viscoelastic properties of lubricating grease containing various thickener concentrations, J. of Coll. and Int. Sci., 274, 285-293, 2004.

YONGGANG, M.; JIE, Z. A rheological model for lithium lubricating grease, Trib. Inter., Vol 31, N 10, pp 619-625, 1998. 